\title{
Molecular simulations of electrolyte structure and dynamics in lithium-sulfur battery solvents
}

\author{
Chanbum Park $^{\mathrm{a}, \mathrm{b}}$, Matej Kanduča ${ }^{\mathrm{a}}$, Richard Chudoba ${ }^{\mathrm{a}, \mathrm{b}}$, Arne Ronneburga ${ }^{\mathrm{a}, \mathrm{b}}$, Sebastian Risse ${ }^{\mathrm{a}}$, Matthias Ballauff ${ }^{\mathrm{a}, \mathrm{b}}$, Joachim \\ Dzubiella $^{\mathrm{a}, \mathrm{b}, *}$ \\ ${ }^{a}$ Institut für Weiche Materie und Funktionale Materialien, Helmholtz-Zentrum Berlin für Materialien und Energie, Hahn-Meitner-Platz. 1, 14109 Berlin, Germany \\ ${ }^{b}$ Institut für Physik, Humboldt-Universität zu Berlin, Newtonstr. 15, 12489 Berlin, Germany
}

\section{Abstract}

The performance of modern lithium-sulfur $(\mathrm{Li} / \mathrm{S})$ battery systems critically depends on the electrolyte and solvent compositions. For fundamental molecular insights and rational guidance of experimental developments, efficient and sufficiently accurate molecular simulations are thus in urgent need. Here, we construct a molecular dynamics (MD) computer simulation model of representative state-of-the art electrolyte-solvent systems for Li/S batteries constituted by lithium-bis(trifluoromethane)sulfonimide in (LiTFSI) and $\mathrm{LiNO}_{3}$ electrolytes in mixtures of the organic solvents 1,2-dimethoxyethane (DME) and 1,3-dioxolane (DOL). We Venchmark and verify our simulations by comparing structural and dynamic features with various available experimental reference systems and demonstrate their applicability for a wide range of electrolyte-solvent compositions. For the state-of-the-art battery solvent, we finally calculate and discuss the detailed composition of the first lithium solvation shell, the temperature dependence of lithium diffusion, as well as the electrolyte conductivities and lithium transference numbers. Our model will serve as a basis for efficient future predictions of electrolyte structure and transport in complex electrode confinements for the optimization of modern $\mathrm{Li} / \mathrm{S}$ batteries (and related devices).

Keywords: Battery, Electrolyte, Molecular Dynamics, Conductivity, Solvation, Ion pairing.

\section{Introduction}

Lithium-sulfur (Li/S) batteries are discussed as a cost efficient key technology for future applications in portable electronic devices, electromobility, and as a backup storage system for the reliable use of renewable energies [1-10]. Because of their high theoretical electrochemical capacity of $1675 \mathrm{~mA} \mathrm{~h} \mathrm{~g}^{-1}, \mathrm{Li} / \mathrm{S}$ batteries represent in principle an efficient energy storage system. Moreover, the abundance and low-cost of their raw materials are important advantages of this battery concept.

The actual performance delivered by $\mathrm{Li} / \mathrm{S}$ batteries is proving to be severely limited in many cases, which is directly related to the role of the electrolyte [6-8, 10-14]. Ultimately, the successful development of the Li/S battery requires careful coordination of the choice of electrolyte with the specific nature of the cathode material. In particular, the optimal electrolyte has to fulfill several boundary conditions, as such to maximize charge carrier conductivity and high ionic dissociation but also to guarantee $\mathrm{Li}^{+}$dissolution and stabilization of the lithium anode [15]. For the latter, the most prominent example is lithium nitrate $\left(\mathrm{LiNO}_{3}\right)$, which has been introduced to stabilize the anode by a protective layer formed on the electrode surface [16, 17]. Recent developments have empirically demonstrated that lithium

\footnotetext{
${ }^{*}$ Corresponding author

Email address: joachim.dzubiella@helmholtz-berlin.de (Joachim Dzubiella)
}

TFSI (bis(trifluoromethane)sulfonimide) salts (at about $1 \mathrm{M}$ concentration) in 1:1 mixtures of the organic solvents 1,2dimethoxyethane (DME) and 1,3-dioxolane (DOL) are found to be a suitable electrolyte solution for $\mathrm{Li} / \mathrm{S}$ batteries, satisfying many of the requirements [7, 18]. More generally, these and similar electrolyte/solvent compositions are also relevant for the development of lithium-oxygen and lithium-air batteries [12, 14, 19, 20] as well as for $\mathrm{Na} / \mathrm{S}$ batteries [21].

For fundamental structural insights on a molecular level and rational guidance of experimental developments, efficient and accurate molecular simulations are of significant importance. For this, classical polarizable or non-polarizable all-atom force field simulations promise the best compromise between accuracy and efficiency [22-35]. For example, they can demonstrate how the details of local solvation structures or ion pairing affinities can be linked to transport properties, such as diffusion and conductivity, i.e., they establish structure-property-function relationships. In particular, they elucidate the effects of organic solvents on the lithium ion solvation and transport in ionic liquid electrolytes [29], i.e., the solvate structures of LiTFSI electrolytes [31, 32] as well as the influence of cations on lithium ion coordination and transport [30]. However, despite their importance for modern $\mathrm{Li} / \mathrm{S}$ battery development, the simulation studies of the structural properties of the lithium salts in mixtures of DME/DOL solvents are scant [76]. Of particular interest is, for instance, an accurate structural characterization of the local lithium solvation structure, which is decisive for ion permeation and capacitance build-up within the commonly used 
porous organic electrode materials.

Here, we construct a molecular dynamics (MD) computer simulation model of representative state-of-the art electrolytesolvent systems [7, 18] for Li/S batteries constituted by LiTFSI and $\mathrm{LiNO}_{3}$ electrolytes in mixtures of DME and DOL. We focus on a force field without explicit many-body polarizability as often used before [33] in order to enhance computing speed and invoke less parameters, aiming at comparable accuracy of structural and dynamic properties. We benchmark and verify our simulations by comparing those calculated structural and dynamic features with various available reference systems, i.e., structure, density, dielectric constant, and viscosity of the organic solvents and their mixtures [28, 36, 38], as well as ionic diffusion and conductivity in dilute electrolytes in the respective pure (one-component) solvents [39]. With these we demonstrate their applicability for a wide range of electrolyte-solvent compositions. As a first practical demonstration at hand of the state-of-the-art electrolyte solvent for $\mathrm{Li} / \mathrm{S}$ batteries, we calculate and discuss the detailed composition of the first lithium solvation shell, the temperature dependence of lithium diffusion, as well as the electrolyte conductivities and lithium transference numbers. We complement this with new experimental measurements on viscosity and conductivity. Our validated model will thus serve as a basis for efficient future predictions of electrolyte structure and transport in complex electrode confinements for the optimization of modern Li/S batteries (and related devices).

This paper is organized as follows: we first describe in detail the used simulation model and underlying analyzing numerical methods for evaluations of relevant quantities. In Section 3 we describe experimental settings. We then present and discuss the results in Section 4, followed by concluding remarks in Section 5

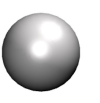

(a)

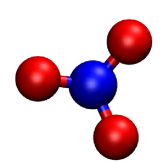

(b)

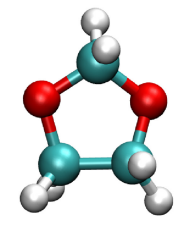

(d)

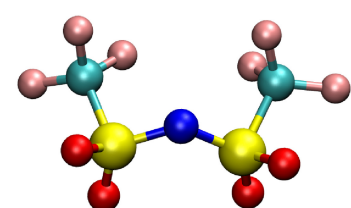

(c)

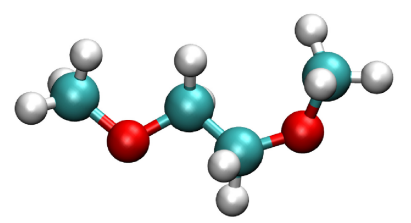

(e)
Fig. 1: Exemplary illustrations of the ions and solvent molecules involved in our simulation study: Ions: (a) $\mathrm{Li}^{+}$, (b) $\mathrm{NO}_{3}{ }^{-}$, (c) $\mathrm{TFSI}^{-}$and organic solvent molecules: (d) DOL and (e) DME. The sizes of $\mathrm{Li}^{+}$and $\mathrm{NO}_{3}{ }^{-}$are scaled up for aesthetic reason.

\section{Computer simulations and analysis methods}

\subsection{Simulation details}

We perform all-atom MD computer simulations of bulk electrolytes in mixed organic solvents constituted of the molecules displayed in Fig. 11 employing the GROMACS 5.1 simulation package [40]. The production simulations are performed in the $N p T$ ensemble at constant pressure and constant temperature in a cubic box with periodic boundary conditions in all three Cartesian directions. The temperature is maintained by the Berendsen thermostat at $304 \mathrm{~K}$ in system III (defined further below) and $298 \mathrm{~K}$ for all other systems with a time constant of $0.1 \mathrm{ps}$ [41]. A constant pressure of 1 bar is controlled by the Parrinello-Rahman barostat [42, 43] with a coupling constant of 2 ps. Electrostatic interactions are treated using the Particle-Mesh-Ewald (PME) method [44, 45] with the Fourier spacing of $0.12 \mathrm{~nm}$ and $1 \mathrm{~nm}$ real-space cut-off. For non-neutral systems, we apply uniform neutralizing background charge in the PME. The molecules are represented by non-polarizable models, i.e., explicit electronic polarization effects are neglected. We consider the polarization implicitly as discussed in the force-field subsection below. All nonbonded non-electrostatic interatomic interactions are based on the Lennard-Jones ( $\mathrm{LJ})$ potential with a cut-off at $1 \mathrm{~nm}$ and shifted to zero there, together with the geometric combination rules [27] $\sigma_{i j}=\left(\sigma_{i i} \sigma_{j j}\right)^{1 / 2}$ and $\epsilon_{i j}=\left(\epsilon_{i i} \epsilon_{j j}\right)^{1 / 2}$ for the LJ size and energy parameters, respectively. The LINCS algorithm [46, 47] is employed for all bond constraints. The integration time step is $2 \mathrm{fs}$. In order to facilitate the equilibriation of solvent conformers, we first perform simulated annealing approach, where we heat the systems to $440 \mathrm{~K}$ or $500 \mathrm{~K}$ and then cool them down to $298 \mathrm{~K}$ on a time interval of $2 \mathrm{~ns}$ in the $N V T$ ensemble.

\subsection{Force fields}

First we have scrutinized the properties of pure solvents of DME and DOL using various force fields and compared them with experimental benchmarks in terms of density, dielectric constant, and viscosity. The properties of DOL were assessed with three different force fields: AMBER [80], the Transferable Potentials for Phase Equilibria united-atom force field (TraPPE) [26], and the Optimized Parameters for Liquid Simulations all-atom force-field (OPLS-AA) [27]. For DME, only AMBER and OPLS-AA force fields were applicable. For DME, instead of the standard dihedrals in the latter two forcefields, we implemented the optimized dihedral parameters as suggested by Anderson et al. [28] based on the comparison with experimental measurements of the molecular conformation populations. The torsional degrees of freedom lead to the occurrence of many different equilibrium conformers and thus significantly affect the instantaneous molecular multipole. The reproduction of the correct mean static dielectric constant of DME solutions is therefore very challenging for computer simulations. We have found that OPLS-AA (with the improved dihedrals for DME) shows consistently the best overall performance for both solvents as discussed below in the Results section. In the Supplementary Information (SI) in Table S1 we 
summarize the MD simulations results for the solvents from all tested force fields.

For low dielectric solvents DOL and DME, extra care has to be taken how to include electronic polarizability effects, which account already for more than $20 \%$ of the total static permittivity and thus cannot be neglected. In view of large scale future applications, such as solvent in electrode confinement, we want to avoid introducing additional parameters into the model as well as increased intensity of the simulations. We therefore opt for an implicit inclusion of electronic (high-frequency) polarizability contribution by the Molecular Dynamics Electronic Continuum (MDEC) model [48-50]. It includes the electronic polarizability implicitly by replacing all partial charges $q_{i}$ of ions in the simulations by effective, rescaled, charges $q_{i}^{\text {eff }}$, according to

$$
q_{i}^{\mathrm{eff}}=\frac{q_{i}}{\sqrt{\epsilon_{\infty}}}
$$

Here, $\epsilon_{\infty}$ is the high-frequency contribution to the solvent permittivity stemming from electronic fluctuations in the solvent molecules [51]. It can be related to the refractive index $n$ as $\epsilon_{\infty}=n^{2}$. From the refractive indices $n$ of 1.3781 for DME and 1.3992 for DOL [51], we obtain an effective charge of a monovalent ion of 0.73 in DME and 0.71 in DOL using eq. (1). We further assume that the effective charge in a mixture of DME and DOL is given simply via a linear interpolation between the effective charges in the pure DME and the pure DOL solutions. We apply eq. (1) to all the partial charges of the three considered ions in our study, $\mathrm{Li}^{+}, \mathrm{NO}_{3}{ }^{-}$, and $\mathrm{TFSI}^{-}$. The force-fields parameters of $\mathrm{TFSI}^{-}$and $\mathrm{NO}_{3}{ }^{-}$are taken from Refs. [52, 53]. On the case of $\mathrm{Li}^{+}$, we have tested various established $\mathrm{LJ}$ parameters and compared them with experimentally available diffusion coefficients of dilute LiTFSI electrolyte in pure DME and DOL solvents (later defined as system III) [39]. While we found that the spread among the performance of the various force fields for the lithium ion is small, i.e., within $15 \%$ for the diffusion coefficient, the best overall performance in combination with the opted anionic force-fields was exhibited by the lithium force field by Dang et al. [54]. Hence the latter has been finally chosen for all our investigated systems with rescaled charges as defined above (see Tables S2 and S3).

\subsection{Simulated systems}

We simulate eight different solution 'systems', denoted in the following as systems I, IIa, IIb, IIc, IIIa, IIIb, IVa, and IVb with the particular number of ions and molecules in the simulation box summarized in Table 1. System I does not include ions and consists only of a reference binary mixture of DME and DOL of varying composition. We express the organic solvent composition as the molar fraction $x$ of DOL in the solvent,

$$
x=\frac{N_{\mathrm{DOL}}}{N_{\mathrm{DOL}}+N_{\mathrm{DME}}},
$$

where $N_{\text {DOL }}$ and $N_{\text {DME }}$ correspond to the number of DOL and DME molecules, respectively. In the system class II we additionally include one $\mathrm{Li}^{+}$(IIa), or one $\mathrm{Li}^{+}-\mathrm{NO}_{3}{ }^{-}$pair (IIb), or one $\mathrm{Li}^{+}-\mathrm{TFSI}^{-}$pair (IIc) to investigate diffusion and structural properties in the high dilution limit of electrolyte, also for various ratios $x=0$ to 1 . Systems IIIa and IIIb relate to an experimental study where diffusion coefficients and conductivity were accurately measured [39] and consists of $25 \mathrm{Li}^{+}-\mathrm{TFSI}^{-}$ ion pairs in either $500 \mathrm{DME}$ or 500 DOL solvent molecules, respectively. The molar ratio between salt and solvent is thus 1:20 in this system. Finally, systems IVa and IVb represent experimental state-of-the-art compositions for a few modern batteries under development and consider concentrated electrolyte mixtures of $\mathrm{Li}^{+}, \mathrm{NO}_{3}{ }^{-}$, and $\mathrm{TFSI}^{-}$at molar concentrations, given in Table 1. System IVb has a similar ionic strength as system IVa but contains no nitrate ions.

Table 1: Numbers of ions and solvent molecules (i.e., the composition) in the investigated simulation systems. System I does not contain ions and the molar ratio $x=N_{\mathrm{DOL}} /\left(N_{\mathrm{DOL}}+N_{\mathrm{DME}}\right)$ of DME/DOL is varied between 0 and 1 with a total number of 508 solvent molecules. System class II has only one $\mathrm{Li}^{+}$ion (IIa) or one ion pair (IIb and IIc) for various DME/DOL ratios. System class III features a 1:20 LiTFSI molar ratio in either DME (IIIa) or DOL (IIIb). Systems IVa and $b$ are representative experimental state-of-the-art systems [7] 18] for $\mathrm{Li} / \mathrm{S}$ batteries with a molar composition of (a) $0.66 \mathrm{M} \mathrm{LiNO}_{3}, 0.33 \mathrm{M}$ LiTFSI, 4.94 M DME, and 6.03 M DOL [55] and (b) 0.88 M LiTFSI, 4.64 M DME, and 5.67 M DOL [56].

\begin{tabular}{|c|c|c|c|c|}
\hline System & $\mathrm{Li}^{+}$ & $\mathrm{NO}_{3}{ }^{-}$ & TFSI $^{-}$ & solvent (DME / DOL) \\
\hline I & & & & $0 . .508 / 0 . .508$ \\
\hline IIa & 1 & & & $0 . .508 / 0 . .508$ \\
\hline IIb & 1 & 1 & & $0 . .508 / 0 . .508$ \\
\hline IIc & 1 & & 1 & $0 . .508 / 0 . .508$ \\
\hline IIIa & 25 & & 25 & $500 / 0$ \\
\hline IIIb & 25 & & 25 & $0 / 500$ \\
\hline IVa & 90 & 60 & 30 & $450 / 550$ \\
\hline IVb & 85 & & 85 & $450 / 550$ \\
\hline
\end{tabular}

\subsection{Analysis}

\subsubsection{Dielectric constant}

The non-electronic part of the static dielectric constant in the simulations is computed based on fluctuations of the Neumann dipole moment $\mathbf{M}$ of the system, which is the sum of all molecular dipole moments $\mathbf{M}=\Sigma_{i} \boldsymbol{\mu}_{i}$ [57-59]. Since we are employing the MDEC treatment in our simulations, where the ionic charges are rescaled via eq. (1), the total dipole is $\mathbf{M}=\mathbf{M}_{\mathrm{MD}} \sqrt{\epsilon_{\infty}}$, where $\mathbf{M}_{\mathrm{MD}}$ is the dipole moment obtained from the simulations. The dielectric constant $\epsilon_{\mathrm{MD}}$ is calculated via [60]

$$
\epsilon_{\mathrm{MD}}=1+\frac{4 \pi}{3 V k_{\mathrm{B}} T}\left(\left\langle\mathbf{M}_{\mathrm{MD}}^{2}\right\rangle-\left\langle\mathbf{M}_{\mathrm{MD}}\right\rangle^{2}\right)
$$

where $V, k_{\mathrm{B}}, T$ are the volume of the simulation box, the Boltzmann constant, and the absolute temperature, respectively. The dielectric constant can be affected by the size of the simulation box [79]. We have verified that the smallest simulation box size of our system is large enough, such that the dielectric constant is not affected by the finite-size effects (see Fig. S1). Finally, we 
calculate the total static dielectric constant $\epsilon$ for the comparison to experimental reality as [49, 50, 57, 61]

$$
\epsilon=\epsilon_{\infty} \epsilon_{\mathrm{MD}}
$$

\subsubsection{Viscosity}

The shear viscosity $\eta_{\infty}$ is calculated from transverse current correlation function [62 64], using the transverse momentum fields (transverse-current autocorrelation function) as implemented in the GROMACS simulation package [40]. Here, a total 16 transverse-current autocorrelation functions corresponding to different $k$-vectors are considered, resulting in 16 values of $\eta$. The values of $\eta$ are fitted to $\eta(k)=\eta_{\infty}\left(1-a k^{2}\right)$, yielding the shear viscosity $\eta_{\infty}$.

In cases of binary solvent mixtures, as we investigate here, we compare the results for the viscosity to the values obtained via well-established semi-empirical analytical mixing rules by Fort and Moore [65] for the experimentally expected viscosity. There, the viscosity $\eta_{\text {mix }}$ of a mixture is calculated from the viscosities of the pure components $\eta_{\infty, 1}$ and $\eta_{\infty, 2}$ as

$$
\eta_{\text {mix }}\left(\phi_{1}, \phi_{2}\right)=\eta_{\infty, 1}^{\phi_{1}} \eta_{\infty, 2}^{\phi_{2}}
$$

The volume fractions $\phi_{1}$ and $\phi_{2}$ of each of the components, $\phi_{i}=V_{i} /\left(V_{1}+V_{2}\right)$, are obtained from the partial volumes $V_{i}$ calculated as $V_{i}=m_{i} / \rho_{i}^{0}$ with $\rho_{i}^{0}$ being the mass density of the pure component.

\subsubsection{Long-time self-diffusion coefficient}

The long-time self-diffusion coefficients of the molecules in the simulations are calculated from the mean square displacement relation,

$$
D_{\mathrm{MD}}=\lim _{\Delta t \rightarrow \infty} \frac{\left\langle r^{2}(\Delta t)\right\rangle}{6 \Delta t} .
$$

Due to finite size effects of a simulation box, hydrodynamic corrections can play a significant role. It has been shown that hydrodynamic perturbations in a small periodic box lead to a finite size correction (FSC) of the diffusion coefficient, $\Delta D_{\mathrm{FSC}}$. In the leading order, $\Delta D_{\mathrm{FSC}}$ scales inversely with the length $L$ of the simulation box and the viscosity $\eta$ of the solution, $\Delta D_{\mathrm{FSC}} \propto 1 /\left(\eta_{\infty} L\right)$ [66]. Although the scaling prefactor of the latter expression can be analytically calculated based on the hydrodynamic self-interaction of a point perturbation, deviations occur for larger and more complex solutes [67]. Therefore, we estimate the correction in our system by simulating the diffusion in boxes of various sizes and fitting its dependence to $\Delta D_{\mathrm{FSC}}=c / L$ with $c$ as a fitting parameter (Figs. S2, S3, and $\mathrm{S} 4$ in the SI). We determine the coefficient $c$ only for the diffusion of the solvent components, DME and DOL, as there the statistical accuracy is highest, and apply the correction also to the diffusion coefficients of ions. The diffusion coefficient we compare to experiments is finally given by

$$
D=D_{\mathrm{MD}}+\Delta D_{\mathrm{FSC}}
$$

\subsubsection{Conductivity and transference number}

The stationary linear response conductivity $\sigma$ is defined by Ohm's law

$$
J=\sigma E,
$$

(expressed here simply as scalar quantities), where $E$ is the external electrostatic driving field and $J=\sum_{i} J_{i}=\sum_{i} \sigma_{i} E$ the sum of all ionic current densities, which define individual partial ionic conductivities $\sigma_{i}$. The transference number of an ion $i$ is defined by [68]

$$
t_{i}=\frac{J_{i}}{J}=\frac{\sigma_{i}}{\sigma}
$$

and describes the relative contribution of the current of species $i$ to the total current. By applying an external field $E$ in the range between 0.005 and $0.05 \mathrm{~V} \mathrm{~nm}^{-1}$ (for which we verified to lie within the linear response regime, see SI Figs. S5 and S6) and calculating resulting individual currents $J_{i}$, we obtain partial conductivities $\sigma_{i}$ via eq. (8). By measuring the mean drift velocities $v_{i}$ of each ionic species under the external field, we calculate the current densities as $J_{i}=z_{i} e n_{i} v_{i}$, where $n_{i}$ is the ionic number density and $z_{i}$ and $e$ the valency and the elementary charge, respectively. In the ideal ion limit one expects the Nernst-Einstein relation to be applicable. It relates the total conductivity and diffusion coefficients, $\sigma^{\mathrm{id}}=\sum_{i}\left|z_{i}\right| e D_{i} n_{i} / k_{\mathrm{B}} T$, and thus gives a reference to conductivity values regarding ideal ionic transport behavior. A degree of ion uncorrelated motion can be defined by $\alpha=\sigma / \sigma^{\text {id }}$. It was shown that the parameter $\alpha$ is tightly related to ion-pair formations [34], whereby smaller values imply higher degree of pair formation.

\subsubsection{Coordination number}

The coordination number of molecules of type $i$ in the first solvation shell surrounding a single molecule of type $j$ is calculated as

$$
N_{i}=4 \pi n_{j} \int_{0}^{R_{\mathrm{M}}} g_{i j}(r) r^{2} \mathrm{~d} r
$$

where $R_{\mathrm{M}}$ is the distance of the first minimum following the first peak in the radial distribution function $(\mathrm{RDF}), g_{i j}(r)$, which is a standard approach for bulk liquids [69].

\section{Experimental characterization of system IVa}

The gravimetric density of the samples was measured at $28^{\circ} \mathrm{C}$ and at a pressure of 1.0186 bar using a chempro/PAAR DMA 602 density meter with a Julabo F25 thermostat. The average value of ten measurements of the natural frequency of a glass tube filled with the solution was taken to calculate the density. Millipore water and air served as reference for this calculation. The viscosity was determined using a Capillary Viscometer (SI Analytics 50101/0a) and a laboratory stopwatch. The viscosity was averaged over three measurements that were performed in an argon filled glovebox. The conductivity was evaluated by performing an impedance spectroscopy in the frequency range of $100 \mathrm{mHz}$ to $1 \mathrm{MHz}$ with $5 \mathrm{mV}$ RMS voltage signal and 15 points per decade. A GAMRY interface 1000 potentiostat and an in-house-designed electrochemical cell were 
used. The cell consists of aluminum-electrodes and a cell housing made of PEEK (Polyether Ether Ketone). The electrode distance is $1 \mathrm{~mm}$, the circular electrode area is $198.56 \mathrm{~mm}^{2}$ (15.9 $\mathrm{mm}$ in diameter). The electrolyte was filled in through a hole on the side of the PEEK cell housing to ensure complete filling. The conductivity was determined by the intersection of the impedance with the $\mathrm{x}$-axis at high frequencies in a Nyquistplot.

\section{Results and discussion}

\subsection{System I : Pure solvent (DME/DOL) mixtures}

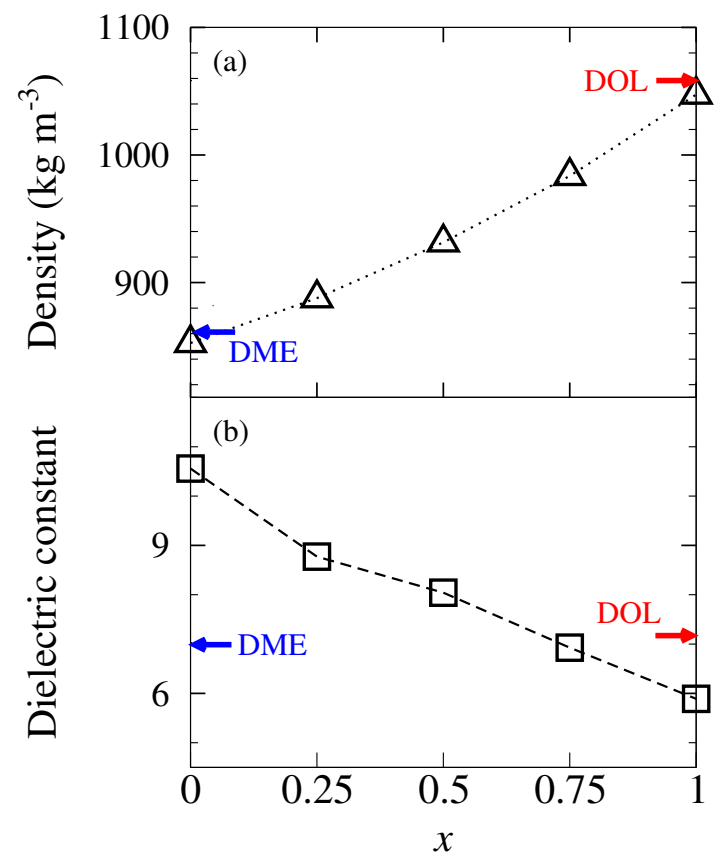

Fig. 2: (a) Density of the DME/DOL mixture (system I) versus the molar fraction of DOL composition $x=N_{\mathrm{DOL}} /\left(N_{\mathrm{DOL}}+N_{\mathrm{DME}}\right)$ from our MD simulations (triangular symbols). (b) Dielectric constant (square symbols) from the MD, eq. (4), for the same systems as in (a). The colored arrows indicate the experimental reference values of pure DME [37] and pure DOL [38], respectively.

The density and dielectric constant of the DME/DOL mixtures as a function of the molar fraction $x$ of DOL are shown in Fig. 2.a) and (b), respectively. The density of the pure DME (i.e., $x=0$ ) obtained from $\mathrm{MD}$ is $853 \mathrm{~kg} \mathrm{~m}^{-3}$, which very well agrees with the experimental value of $861 \mathrm{~kg} \mathrm{~m}^{-3}$ [37]. Also the density of the pure DOL (i.e., $x=1$ ) from MD, $1047 \mathrm{~kg} \mathrm{~m}^{-3}$, is in good agreement with the experimental one, $1059 \mathrm{~kg} \mathrm{~m}^{-3}$ [38]. The density of the mixture increases monotonically with the molar fraction $x$ of DOL. Turning to the dielectric constant in panel (b), we find satisfactory agreement for the pure DOL solvent at $x=1$, where the simulated value is about $16 \%$ smaller than in the experiments. The MD value for the pure DME $(x=0)$ is less satisfying and with 10.6 compared with the experimental 7.1 almost $50 \%$ too large. However, after having examined and thoroughly scrutinized various force field combinations, we found this deviation still to be minimal under the constraint that the density as well as the viscosity (shown below) reproduce well the experimental reality.

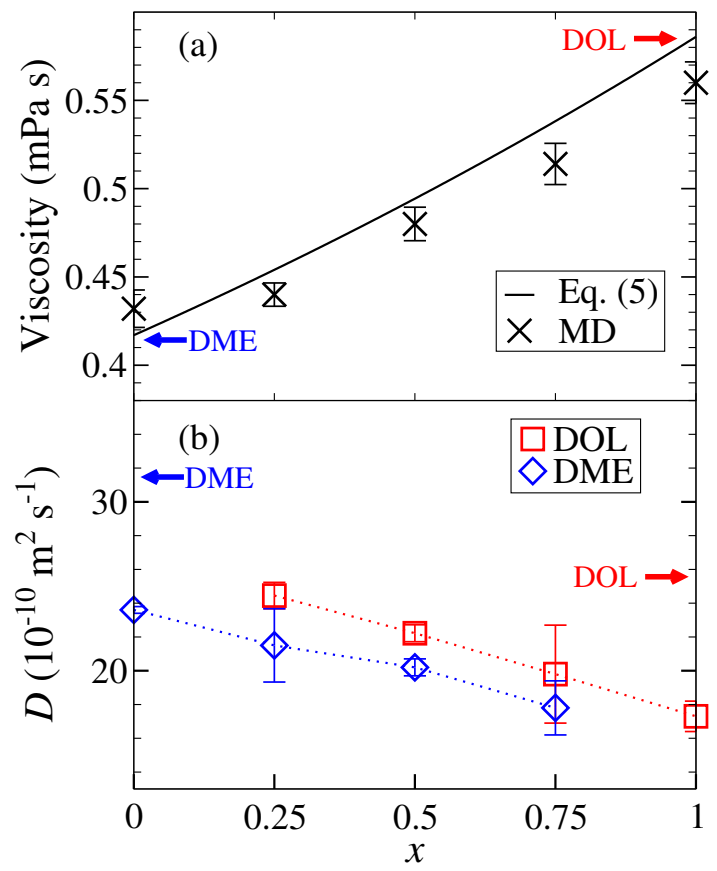

Fig. 3: (a) Shear viscosity $\eta(x)$ of the binary DME/DOL mixtures versus the molar fraction of DOL $x$. Crosses indicate the results obtained from the MD simulations and the solid line represents the viscosity of the binary mixture from eq. 5, which interpolates the experimental limits of the pure DME [37] and pure DOL [38], indicated by arrows. (b) Self-diffusion coefficients of DME molecules (diamonds) and DOL molecules (squares) in the DME/DOL mixture fas a function of the DOL molar fraction $x$. The experimental reference values of DME and DOL [39] are indicated by a blue and a red arrow, respectively.

The viscosity of the DME/DOL mixtures from our simulations is presented in Fig. 3 a). There, we also plot experimentally measured viscosities or pure solvents and apply the analytical mixing rule eq. (5) for the mixtures. We see that the simulations for the pure DME and DOL systems yield viscosities of $0.43 \mathrm{mPas}$ and $0.56 \mathrm{mPas}$, respectively, which compare well with the experimental ones of $0.42 \mathrm{mPas}$ and $0.58 \mathrm{mPa}$ [ [37, 38], as well as with the expected interpolation behavior, eq. (5). As another important transport property we have calculated the self-diffusion coefficients of the solvent molecules in the mixtures, cf. panel (b) of the same figure. The simulations, corrected for finite-size effects (cf. SI), underestimate the experimental reference values by $27 \%$ (DME) and $32 \%$ (DOL). The interpolation between the limiting cases $x=0$ and $x=1$ transits monotonously. However, as we will see later, the diffusion coefficients compare much better to the experimental values of system IVb (Table 2), performed by a different group.

In essence, we can conclude that transport properties are well captured within the MD model. Overall, we find satisfying behavior of our solvent force field in the sense that it can reproduce reasonably well several experimentally important equilibrium thermodynamic and transport properties for the full molar ratio range $x=0$ to $x=1$ at the same time. 
4.2. Systems IIa, IIb, and IIc: single ions or ion pairs in mixed $D M E / D O L$ solvents

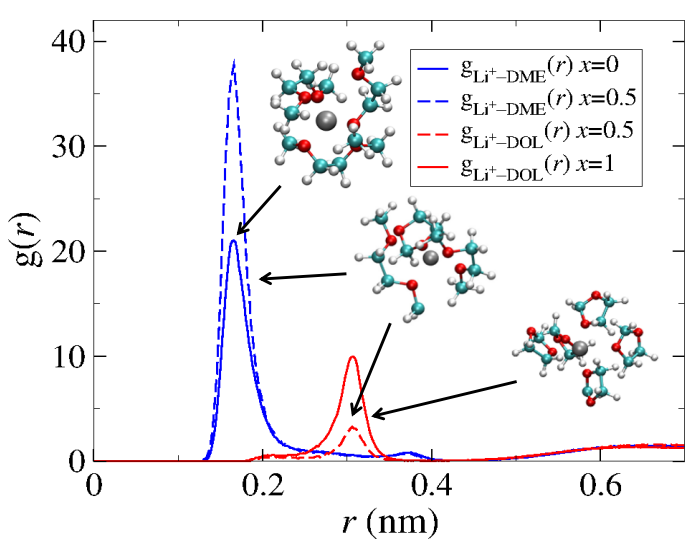

Fig. 4: Center-of-mass RDF between $\mathrm{Li}^{+}$and solvent molecules for system IIa; $g_{\mathrm{Li}^{+}-\mathrm{DME}}(r)$ and $g_{\mathrm{Li}^{+}-\mathrm{DOL}}(r)$ are shown for the limiting cases $x=0$ (DME only) and $x=1$ (DOL only) and for the intermediate ratio $x=0.5$.

Now we consider highly dilute electrolyte solutions, where we investigate the solvation structure and diffusion of a single $\mathrm{Li}^{+}$ion (Sys. IIa), $\mathrm{Li}^{+}-\mathrm{NO}_{3}{ }^{-}$(IIb), and $\mathrm{Li}^{+}-\mathrm{TFSI}^{-}$(IIc) ion pairs in the solvent mixture of system I. In order to discuss the solvation structure, we plot in Fig. 4 the center-of-mass radial distribution function (RDF) between the $\mathrm{Li}^{+}$and solvent molecules in system IIa: $g_{\mathrm{Li}^{+} \text {-DME }}(r)$ and $g_{\mathrm{Li}^{+} \text {-DOL }}(r)$ are shown for the limiting cases $x=0$ (DME only) and $x=1$ (DOL only) and for the intermediate ratio $x=0.5$. The DME distribution peaks at about $0.16 \mathrm{~nm}$ and is thus closer to the $\mathrm{Li}^{+}$ion than DOL molecules, whose distribution peaks at about $0.3 \mathrm{~nm}$. Such a close approach of DME is consistent with experimental data where the $\mathrm{Li}^{+}$-DME coordination leads to cis (the $\mathrm{C}$ $\mathrm{O}$ bonds) and a gauche configurations in DME molecules in a bidentate binding configuration [70, 71]. This is absent for DOL, cf. also the representative simulation snapshots in Fig. 4 Thus, the coordination of DME in the bidentate to $\mathrm{Li}^{+}$ion retains a relatively stable solvation structure even at the symmetric solvent ratio $x=0.5$.

The consequence is an interesting coordination behavior along the mixing coordinate $x$ as presented in Fig. 5.a) for system IIa: let us start at the right hand side of the plot at $x=1$, where DOL coordinates the cation with a coordination number of 4. Adding DME to the solution very quickly substantially changes the DOL coordination; already at around $x \simeq 0.8$ the coordination of DME and DOL equalize (at about 1.8). At a symmetric concentration $(x=0.5)$ the DME is then in large excess with a coordination close to the limiting coordination of about 2.7 of the pure DME $(x=0)$. We note that an analogous 'solvent-exchange' behavior has been observed previously already in DME/propylene carbonate mixtures [72], pointing to the special excess solvation properties of DME in general for its mixtures with other solvents.

For systems IIb and IIc, where also an additional anion is present, corresponding to concentration around $20 \mathrm{mM}$ of the electrolyte, the cation coordination number decreases, but qual-

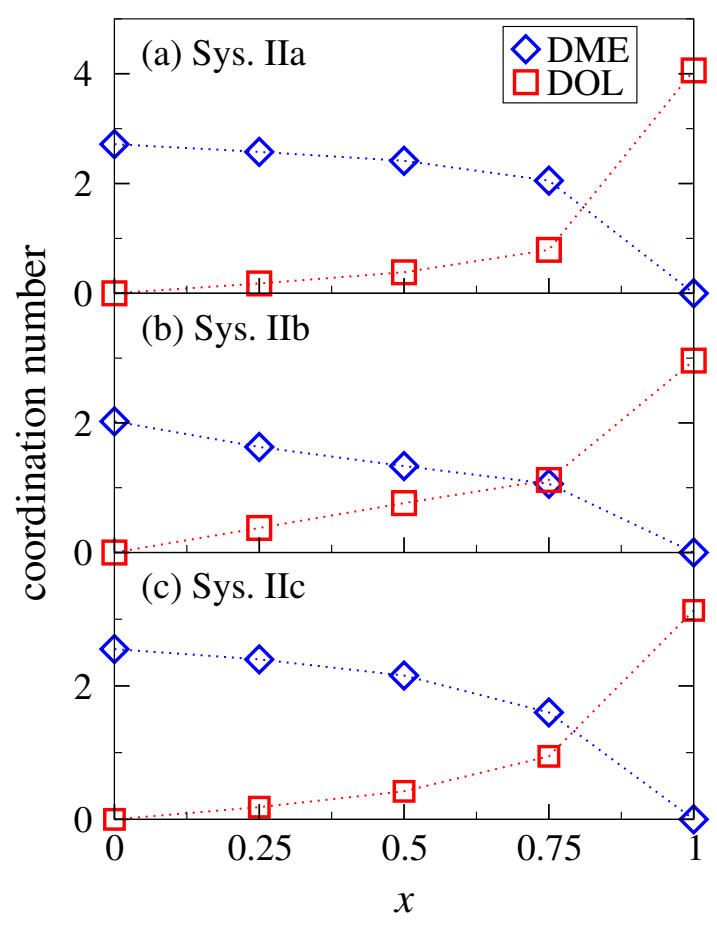

Fig. 5: Coordination number of $\mathrm{Li}^{+}$of DME (blue diamonds) and DOL (red squares) molecules as a function of the molar fraction $x$ in systems IIa, IIb, and IIIc. The dotted connecting lines are plotted as guides to the eye.

itatively retaining the behavior with varying the molar fraction $x$, see Fig. 5(b) and (c). The reason is a strong ion pairing, which is anion-specific. The relatively small $\mathrm{NO}_{3}{ }^{-}$counterion binds very tightly to the $\mathrm{Li}^{+}$cation (see also the discussion later for the concentrated system IVa). The strongly associated $\mathrm{Li}^{+}-\mathrm{NO}_{3}{ }^{-}$ion pairs in the pure DME indeed have been categorized previously already as a 'contact ion pair' (CIP) or even 'aggregate' solvation structure [71]. In this case, the coordination by the organic solvent is consequently reduced by 1 in the whole $x$-range. The larger TFSI $^{-}$anion, however, consistent with the category of a dissociated salt forming 'solventseparated ion pairs' (SSIPs) in pure DME [71], only manages to replace bigger more weakly bound DOL molecules, but not DME molecules. Consequently, the DME coordination around $\mathrm{Li}^{+}$close to $x=0$ remains almost unaffected by the presence of the TFSI ${ }^{-}$ion. The SSIP structure of $\mathrm{Li}^{+}-\mathrm{TFSI}^{-}$can also be empirically explained with the Gutmann donor number (DN) [73, 77, 81], which corresponds to the negative binding enthalpy of a given molecule to a reference Lewis acid. Assuming $\mathrm{Li}^{+}$to behave as a Lewis acid, the DN provides an estimate for the binding affinities of other molecules to $\mathrm{Li}^{+}$. DME has the DN number of 20, while $\mathrm{TFSI}^{-}$the value of 5 . Hence, much weaker association strength of $\mathrm{Li}^{+}-\mathrm{TFSI}^{-}$, as indicated by the smaller DN number, leads to the solvation shell of $\mathrm{Li}^{+}$ dominated by DME (with higher DN). Our results are in line with the reported ionic strength in aprotic solvents in general, where the $\mathrm{TFSI}^{-}$has much smaller association strength than $\mathrm{NO}_{3}{ }^{-}[71]$.

The self-diffusion coefficients of the molecular constituents 
Table 2: Density, dielectric constant, viscosity, $\mathrm{Li}^{+}$coordination, conductivity and diffusion coefficients of systems IIIa (1:20 LiTFSI salt in pure DME), IIIb (1:20 LiTFSI salt in pure DOL), IVa [0.66 $\mathrm{M} \mathrm{LiNO}_{3}$ and 0.33 M LiTFSI in DME:DIOX (45:55 molar ratio)] and IVb [0.88 M LiTFSI in DME:DIOX (45:55 molar ratio)]. Systems IIIa and IIIb are conducted at $T=304 \mathrm{~K}$ and, whereas IVa and IVb at $298 \mathrm{~K}$. Experimental measurements of conductivity in this work (system IVa) are carried out with $0.6 \mathrm{M} \mathrm{LiNO}_{3}$ and $0.3 \mathrm{M}$ LiTFSI in a DME and DOL $(1: 1 \mathrm{wt} \%)$ mixture at room temperature.

\begin{tabular}{|c|c|c|c|c|c|c|c|c|}
\hline & \multicolumn{2}{|c|}{ Sys. IIIa } & \multicolumn{2}{|c|}{ Sys. IIIb } & \multicolumn{2}{|c|}{ Sys. IVa } & \multicolumn{2}{|c|}{ Sys. IVb } \\
\hline & MD & $\begin{array}{l}\text { Exp. } \\
\text { [39] }\end{array}$ & MD & $\begin{array}{l}\text { Exp. } \\
\text { [39] }\end{array}$ & MD & $\begin{array}{c}\text { Exp. } \\
\text { (this work) }\end{array}$ & MD & $\begin{array}{r}\text { Exp. } \\
{[56} \\
74-76 \\
\end{array}$ \\
\hline Density $\left(\mathrm{kg} \mathrm{m}^{-3}\right)$ & $934.1(2)$ & & $1147.0(1)$ & & $1030.0(1)$ & 1103 & $1091.2(1.1)$ & $1125^{a}$ \\
\hline Dielectric constant $\epsilon$ & 8.8 & & 5.7 & & 7.6 & & 6.6 & \\
\hline Viscosity (mPa s) & $0.57(5)$ & & $0.74(5)$ & & $0.77(1)$ & 0.88 & $0.85(3)$ & $\begin{array}{l}1.56^{b}, \\
1.25^{c}\end{array}$ \\
\hline Coordination number of $\mathrm{Li}^{+}$ & 2.45 & & 3.04 & & 3.2 & & 3.2 & \\
\hline Conductivity $\left(\mathrm{S} \mathrm{m}^{-1}\right)$ & $0.73(3)$ & 0.89 & $0.27(3)$ & 0.31 & $0.36(4)$ & $0.59(3)$ & $0.82(3)$ & $\begin{array}{c}1.47^{a} \\
1.32^{b} \\
1.1^{c}\end{array}$ \\
\hline $\begin{array}{l}\text { Degree of ion uncorrelated } \\
\text { motion } \alpha\end{array}$ & 0.28 & 0.31 & 0.1 & 0.1 & 0.12 & & 0.3 & \\
\hline $\mathrm{Li}^{+}$transference $t_{\mathrm{Li}^{+}}$ & 0.4 & 0.47 & 0.5 & 0.51 & 0.61 & & 0.38 & \\
\hline$D_{\mathrm{Li}^{+}}\left(10^{-10} \mathrm{~m}^{2} \mathrm{~s}^{-1}\right)$ & 7.2(1.1) & 7.7 & $5.1(4)$ & 6.4 & $4.0(3)$ & & $4.7(3)$ & $4.3^{d}$ \\
\hline$D_{\mathrm{TFSI}^{-}}\left(10^{-10} \mathrm{~m}^{2} \mathrm{~s}^{-1}\right)$ & $7.8(1.3)$ & 8.8 & $4.5(7)$ & 6.2 & $5.0(1.0)$ & & $3.8(1.0)$ & $4.8^{d}$ \\
\hline$D_{\mathrm{NO}_{3}}-\left(10^{-10} \mathrm{~m}^{2} \mathrm{~s}^{-1}\right)$ & & & & & $3.9(3)$ & & & \\
\hline$D_{\text {DME }}\left(10^{-10} \mathrm{~m}^{2} \mathrm{~s}^{-1}\right)$ & $16.4(1.3)$ & 22.0 & & & $9.9(1.0)$ & & $8.2(1.0)$ & $7.7^{d}$ \\
\hline$D_{\mathrm{DOL}}\left(10^{-10} \mathrm{~m}^{2} \mathrm{~s}^{-1}\right)$ & & & $12.9(4)$ & 17.0 & $13.1(1.0)$ & & $10.0(1.0)$ & $11.4^{d}$ \\
\hline
\end{tabular}

${ }^{a}$ Ref. [56], ${ }^{b}$ Ref. [74], ${ }^{c}$ Ref. [75], and ${ }^{d}$ Ref. [76]

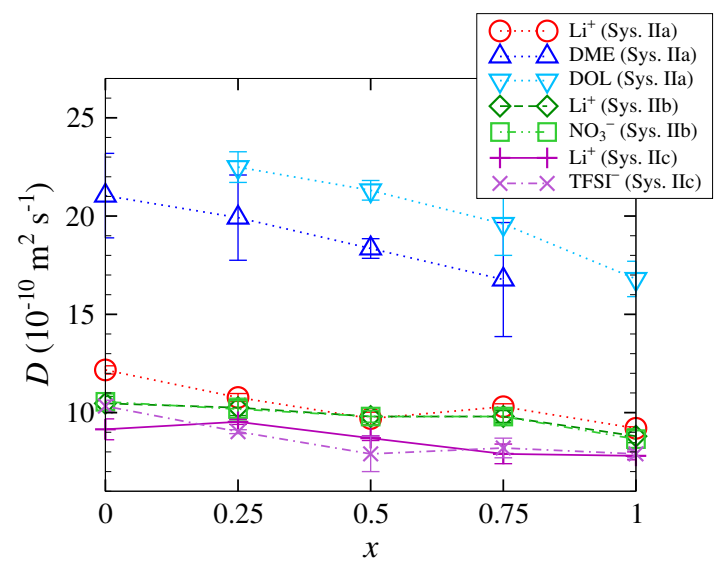

Fig. 6: Diffusion coefficients of $\mathrm{Li}^{+}, \mathrm{NO}_{3}^{-}$, $\mathrm{TFSI}^{-}$, DME, and DOL in systems $\mathrm{IIa}, \mathrm{IIb}$, and IIc as a function of the DOL molar fraction $x$.

in all systems of class II are presented in Fig. 6. In these dilute systems (molar ratio 1:508) the diffusion properties of the pure organic solvent mixtures of DME and DOL are hardly affected. The $\mathrm{Li}^{+}$diffusion coefficient is between $13 \times 10^{-10} \mathrm{~m}^{2} \mathrm{~s}^{-1}$ at $x=0$ and $9 \times 10^{-10} \mathrm{~m}^{2} \mathrm{~s}^{-1}$ at $x=1$ in system IIa, i.e., decreasing with increasing DOL concentration. This effect can be attributed to larger size of the solvation shell in a more coordinated DOL solvent. An inclusion of the counterion has small but visible effects and depends on anion type. For $\mathrm{NO}_{3}{ }^{-}$, a strong ion pair is created, which evidently changes the $\mathrm{Li}^{+}$ diffusion only very slightly although a joint diffusion of the cation-anion pair is apparently established. In the presence of a larger $\mathrm{TFSI}^{-}$anion, the $\mathrm{Li}^{+}$cation diffusion is slowed down more, probably related to a larger size of the formed ion pair, although only existent as an SSIP cluster.

\subsection{Sys. IIIa and IIIb : 1:20 electrolyte to solvent ratio}

Now we investigate the systems with the molar ratio of 1:20 LiTFSI:DME or LiTFSI:DOL, for which accurate experimental data for the conductivity, lithium transference, degree of ion uncorrelated motion, and diffusion coefficients are available [39]. Other physical properties, such as density, dielectric constant, viscosity, and solvent coordination are also calculated and summarized in Table 2 together with the experimental diffusion data. As can be seen, the total conductivity, lithium transference, as well as degree of ion uncorrelated motion are quite well reproduced. The self-diffusion of DME and DOL is in the simulations lower (by about factor of 3/4) than in the experiment. This deviation is consistent with the results of the pure solvent (system I) conducted within the same experimental study [39]. The diffusion coefficients of the $\mathrm{Li}^{+}$and $\mathrm{TFSI}^{-}$ions from the MD also consistently reproduce the experimental trends [39] in both systems IIIa and IIIb. In experiments and simulations, it is observed that the diffusivity in system IIIa is faster than that of IIIb. This can be attributed to the lower viscosity of DME with respect to DOL. Compared with the simulations results of the highly diluted electrolyte in systems II (1:500 ion-solvent ratio), the diffusivities in systems III are all found about $20 \%$ (DME) to $35 \%$ (DOL) lower due to the higher viscosity by the same relative amount. 
The coordination solvent numbers for $\mathrm{Li}^{+}$in systems IIIa and IIIb are 2.45 and 3.04, respectively (see Table 2). The corresponding RDFs (see Figs. S7) indicate, analogously to systems II, that the DME molecule solvates $\mathrm{Li}^{+}$much stronger than DOL. The distance between $\mathrm{Li}^{+}$and the centers-of-mass of DME and $\mathrm{Li}^{+}$and DOL are 0.16 and $0.30 \mathrm{~nm}$, respectively, which is in accord with $g_{\mathrm{Li}^{+}-\mathrm{DME}}(r)$ and $g_{\mathrm{Li}^{+}-\mathrm{DOL}}(r)$ for $x=0$ and $x=1$ in the molar ratio of 1:500 in Sec 4.2 respectively. $g_{\mathrm{TFSI}^{-}-\mathrm{DME}}(r)$ and $g_{\mathrm{TFSI}^{-}-\mathrm{DOL}}(r)$ (see Fig. S8) show the first peak at $0.67 \mathrm{~nm}$ and $0.65 \mathrm{~nm}$, respectively, which are relatively low, similarly as in the much more dilute systems II. This implies that the affinities between $\mathrm{TFSI}^{-}$and DME or DOL are relatively small and do not much depend on the salt concentration.

\subsection{Systems IVa and IVb: Li-ion battery electrolyte solution}

Systems IVa and IVb consider practical Li-ion battery electrolyte solutions with about $0.99 \mathrm{M}$ salt concentration in a 45/55 molar ratio DME/DOL solvent. The individual molar concentrations are $0.66 \mathrm{M} \mathrm{LiNO}_{3}, 0.33 \mathrm{M}$ LiTFSI, 4.94 M DME, and 6.03 M DOL in system IVa [55] and 0.88 M LiTFSI, $4.64 \mathrm{M}$ DME, and 5.67 M DOL in nitrate-free system IVb. Table 2 shows the comparison between MD and experimental results for the density and viscosity, dielectric constant, conductivity and diffusion coefficients for systems IVa and IVb. The MD simulations are able to reproduce well the available experimental values, further verifying the quality of our implemented force field. The MD model reproduces very well all the diffusion coefficients in system IVb (with deviations below $20 \%$ ). Moreover, in system IVa, it yields the conductivity of $0.36 \mathrm{~S} \mathrm{~m}^{-1}$, which is around $40 \%$ lower than the experimental value of $0.59 \mathrm{~S} \mathrm{~m}^{-1}$, but correctly catches the trend of decrease in conductivity due to introduction of $\mathrm{NO}_{3}{ }^{-}$ions (compare systems IVa, IVb; experimental conductivity measurements at different $\mathrm{LiNO}_{3}$ molar concentrations are available in Table S4).

The value of the transference number of the $\mathrm{Li}^{+}$in system IVa is about 0.6. The relatively low value of $\alpha \simeq 0.12$, implyies that strong ion pairing takes place. Namely, the affinity between $\mathrm{Li}^{+}-\mathrm{NO}_{3}{ }^{-}$ion pairs (CIP) in system IVa is large, resulting in correlated diffusion, meanwhile, $\mathrm{Li}^{+}$and $\mathrm{TFSI}^{-}$ions in system IVb are well separated by solvents and contribute to higher conductivity than in system IVa. Since the MD conductivities are significantly lower than the experimental values, it seems that ion pairing is a bit overestimated in our simulations. The conductivity from experimental measurements also show a decrease as $\mathrm{LiNO}_{3}$ molar concentration increases (Table S4). This all are clear signatures of the fact that ionic conductivity is affected by not only ionic strength but also ion-specific pairing.

We are now in the position to interpret and predict interesting properties of these state-of-the-art battery electrolytes and how they depend, for example, on the solvent composition or temperature. Furthermore, we can also obtain a deeper microscopic insight into structural details, e.g., the detailed composition of the first solvation shell of lithium. Hence, in the following, we concentrate exemplarily on the self-diffusion coefficient of the molecules and their temperature dependence and discuss structural features of the first $\mathrm{Li}^{+}$solvation shell in system IVa.
Table 3: Fitting parameters $\ln D_{0}$ and $\Delta E_{\mathrm{a}}$ according to the Arrhenius law for the self-diffusion coefficients in system IVa.

\begin{tabular}{|c|c|c|}
\hline Molecules & $\ln D_{0}$ & $\Delta E_{\mathrm{a}}\left(\mathrm{kJ} \mathrm{mol}^{-1}\right)$ \\
\hline $\mathrm{Li}^{+}$ & $6.3(3)$ & $12.1(1)$ \\
$\mathrm{NO}_{3}{ }^{-}$ & $6.0(3)$ & $11.3(1)$ \\
$\mathrm{TFSI}^{-}$ & $7.4(3)$ & $14.5(2)$ \\
$\mathrm{DME}$ & $7.5(3)$ & $12.8(1)$ \\
$\mathrm{DOL}$ & $7.7(3)$ & $12.8(1)$ \\
\hline
\end{tabular}

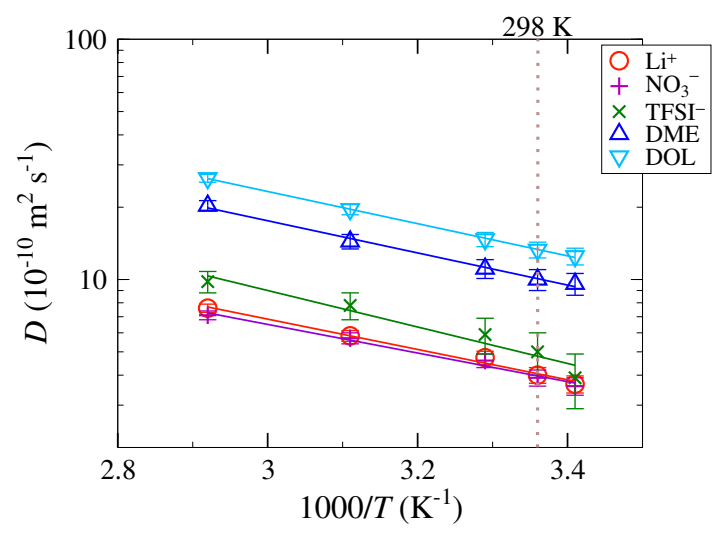

Fig. 7: Diffusion coefficients of ions and solvent molecules in system IVa as a function of inverse temperature in a log-lin representation. They all obey the Arrhenius behavior given by eq. [11. The vertical dotted line indicates $T=298 \mathrm{~K}$

Table 2 (bottom) also summarizes the results for the individual self-diffusion coefficients in system IVa. Compared with the dilute electrolyte systems II and III, the diffusion coefficients of both ions and solvent are substantially lower. Overall, this is a consequence of the higher viscosity of system IVa and ion pairing. The temperature-dependence of ionic diffusion is presented in Fig. 7. which shows an Arrhenius plot for the diffusion coefficients according to the standard law

$$
D(T)=D_{0} \exp \left(-\frac{\Delta E_{\mathrm{a}}}{k_{\mathrm{B}} T}\right),
$$

where $\Delta E_{\mathrm{a}}$ is the activation energy for diffusion. The individual fitting parameters $\ln D_{0}$ and $\Delta E_{\mathrm{a}}$ are summarized in Table 3 . As can be seen, the diffusion (i.e., ionic mobilities in these experimentally relevant systems) can increase almost by a factor 2 or 3 when going from room temperature to relatively hot operating temperatures close to the solvent boiling temperatures $(358 \mathrm{~K}$ for DME and $347 \mathrm{~K}$ for DOL).

We finally turn to the structural description of system IVa. A snapshot of a representative configuration in the first solvation shell of $\mathrm{Li}^{+}$is shown in Fig. 8(a). The RDFs between the center-of-mass of the individual anions and solvent molecules around $\mathrm{Li}^{+}$ion are presented in Fig. 8(b) in a log-lin representation. A distinct solvent composition and layering within the first solvation shell is exhibited. At closest distance is the DME 


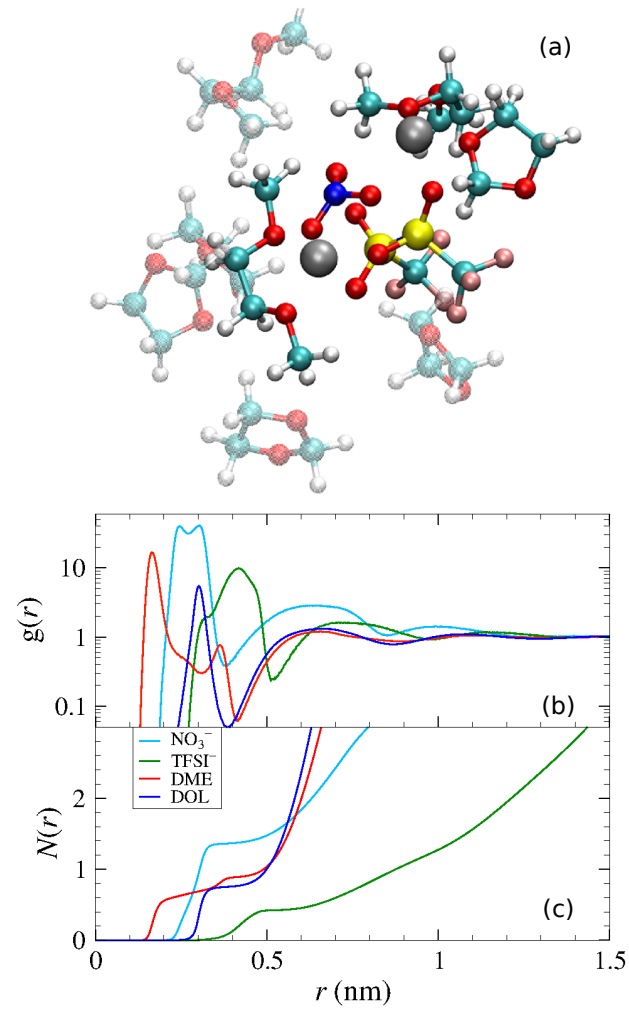

Fig. 8: (a) Simulation snapshot of molecules surrounding $\mathrm{Li}^{+}$ions (gray spheres) in their first solvation shell in system IVa. (b) Center-of-mass RDF and (c) the coordination number $N(r)$ of ions and solvent molecules around a single $\mathrm{Li}^{+}$ion as a function of distance $r$ in system IVa. Note the log-lin presentation in panel (b).

solvent at about $0.165 \mathrm{~nm}$, followed by a large nitrate peak at about $0.25-0.3 \mathrm{~nm}$ and the DOL at about $0.3 \mathrm{~nm}$. The large TFSI $^{-}$has its center-of-mass a bit more outwards, peaking at about $0.42 \mathrm{~nm}$. The first solvation shell according to these distributions has a radius of about $0.5 \mathrm{~nm}$ (see also Figs S9). This running coordination number of the molecules around the $\mathrm{Li}^{+}$ ion is displayed in panel (c) of the same figure. The coordination numbers in the first solvation shell (i.e., within $\simeq 0.5 \mathrm{~nm}$ ) are about 1.1 for DME, 0.7 for DOL, 1.3 for $\mathrm{NO}_{3}{ }^{-}$, and 0.4 for $\mathrm{TFSI}^{-}$, on average. Altogether this makes 3.2 molecules in the first solvation shell. Most qualitative structural features of system IVa thus resemble those of the dilute systems discussed before (see also Figs. S9 which, for instance, exhibit a similar structure of RDFs between system IIa and IVa).

\section{Summary and concluding remarks}

In this work, we constructed an efficient molecular model for state-of-the-art Li/S battery electrolytes and solvents that reproduces a variety of experimentally observable structural and dynamical features. We validated it with various reference systems at hand, in particular in those limits where neat experimental data were available. For example, the density, dielectric constant, viscosity, and diffusion coefficient of solvent mixtures DME/DOL are satisfactorily reproduced for all mo- lar ratios. The $\mathrm{Li}^{+}$solvation structure and pair association with $\mathrm{NO}_{3}{ }^{-}$and $\mathrm{TFSI}^{-}$anions in DME were found consistent with experimental data. The ion mobility and conductivity in 1:20 salt-solvent systems as well agreed with experimental measurements. Finally, the physical properties, such as $\mathrm{Li}^{+}$solvation environment and diffusivity of the full state-of-the-art Li/S battery electrolytes were in detail investigated and gave unprecedented structural insight in the composition of the important first solvation shell of the $\mathrm{Li}^{+}$ion. Apart from the fundamental insights provided, our model will thus serve as a basis for efficient future modelings of electrolyte structure, conductivity, capacity, etc. in various electrolyte solvent compositions in porous electrode confinements and interfaces. With that, it will provide a guidance for the development of modern $\mathrm{Li} / \mathrm{S}$ batteries and related systems.

\section{Acknowledgments}

The authors thank Won Kyu Kim, Rafael Roa Chamorro, and R. Gregor Weiß for useful discussions.

\section{References}

[1] A. Rosenman, E. Markevich, G. Salitra, D. Aurbach, A. Garsuch, F. F. Chesneau, Review on Li-sulfur battery systems: an integral perspective, Adv. Energy Mater. 5 (16).

[2] F. Schipper, D. Aurbach, A brief review: Past, present and future of lithium ion batteries, Russ. J. Electrochem. 52 (12) (2016) 1095-1121.

[3] M. Cuisinier, C. Hart, M. Balasubramanian, A. Garsuch, L. F. Nazar, Radical or not radical: revisiting lithium-sulfur electrochemistry in nonaqueous electrolytes, Adv. Energy Mater. 5 (16).

[4] X. Ji, K. T. Lee, L. F. Nazar, A highly ordered nanostructured carbonsulphur cathode for lithium-sulphur batteries, Nat. Mater. 8 (6) (2009) 500-506.

[5] X. Ji, L. F. Nazar, Advances in Li-S batteries, J. Mater. Chem. 20 (44) (2010) 9821-9826.

[6] J. Gao, M. A. Lowe, Y. Kiya, H. D. Abruña, Effects of liquid electrolytes on the charge-discharge performance of rechargeable lithium/sulfur batteries: electrochemical and in-situ x-ray absorption spectroscopic studies, J. Phys. Chem. C 115 (50) (2011) 25132-25137.

[7] J. Scheers, S. Fantini, P. Johansson, A review of electrolytes for lithiumsulphur batteries, J. Power Sources 255 (2014) 204-218.

[8] E. S. Shin, K. Kim, S. H. Oh, W. I. Cho, Polysulfide dissolution control: the common ion effect, Chem. Commun. 49 (20) (2013) 2004-2006.

[9] Y.-X. Yin, S. Xin, Y.-G. Guo, L. Wan, Lithium-sulfur batteries: Electrochemistry, materials, and prospects, Angew. Chem. (Intern. Ed.) 52 (2013) 13186-13200.

[10] M. Vijayakumar, N. Govind, E. Walter, S. D. Burton, A. Shukla, A. Devaraj, J. Xiao, J. Liu, C. Wang, A. Karim, et al., Molecular structure and stability of dissolved lithium polysulfide species, Phys. Chem. Chem. Phys. 16 (22) (2014) 10923-10932.

[11] M. Barghamadi, A. S. Best, A. I. Bhatt, A. F. Hollenkamp, M. Musameh, R. J. Rees, T. Rüther, Lithium-sulfur batteries-the solution is in the electrolyte, but is the electrolyte a solution?, Energy \& Environ Sci. 7 (1) (2014) 3902.

[12] Q. Li, J. Chen, L. Fan, X. Kong, Y. Lu, Progress in electrolytes for rechargeable Li-based batteries and beyond, Green Energy \& Environment 1 (1) (2016) 18-42.

[13] K. Xu, Nonaqueous liquid electrolytes for lithium-based rechargeable batteries, Chem. Rev. 104 (10) (2004) 4303-4418.

[14] F. Li, T. Zhang, H. Zhou, Challenges of non-aqueous $\mathrm{Li}-\mathrm{O}_{2}$ batteries: electrolytes, catalysts, and anodes, Energy Environ Sci 6 (4) (2013) 11251141.

[15] L. Suo, Y.-S. Hu, H. Li, M. Armand, L. Chen, A new class of solventin-salt electrolyte for high-energy rechargeable metallic lithium batteries, Nat. Commun. 4 (2013) 1481. 
[16] Y. V. Mikhaylik, Electrolytes for lithium sulfur cells, US Patent 8,828,610 (Sep. 9 2014)

[17] D. Aurbach, E. Pollak, R. Elazari, G. Salitra, C. S. Kelley, J. Affinito, On the surface chemical aspects of very high energy density, rechargeable Li-sulfur batteries, J. Electrochem. Soc. 156 (8) (2009) A694-A702.

[18] S. Risse, C. J. Jafta, Y. Yang, N. Kardjilov, A. Hilger, I. Manke, M. Ballauff, Multidimensional operando analysis of macroscopic structure evolution in lithium sulfur cells by $\mathrm{x}$-ray radiography, Phys. Chem. Chem. Phys. 18 (2016) 10630-10636.

[19] D. Geng, N. Ding, T. S. A. Hor, S. W. Chien, Z. Liu, D. Wuu, X. Sun, Y. Zong, From Lithium-Oxygen to Lithium-Air batteries: Challenges and Opportunities, Adv. Energy Mater. 6 (2016) 1502164.

[20] M. Balaish, A. Kraytsberg, Y. Ein-Eli, A critical review on lithium-air battery electrolytes, Phys. Chem. Chem. Phys. 16 (2014) 2801.

[21] S. Wenzel, H. Metelmann, C. Raiß, A. Dürr, J. Janek, P. Adelhelm, Thermodynamics and cell chemistry of room temperature sodium/sulfur cells with liquid and liquid/solid electrolyte, J. Power Sources 243 (2013) 758765.

[22] G. D. Smith, R. L. Jaffe, D. Y. Yoon, Force field for simulations of 1, 2 dimethoxyethane and poly (oxyethylene) based upon $a b$ initio electronic structure calculations on model molecules, J. Phys. Chem. 97 (1993) 12752-12759.

[23] D. Bedrov, O. Borodin, G. D. Smith, Molecular dynamics simulations of 1, 2-dimethoxyethane/water solutions. 1. conformational and structural properties, J. Phys. Chem. B 102 (1998) 5683-5690.

[24] J.-C. Soetens, C. Millot, B. Maigret, Molecular dynamics simulation of $\mathrm{Li}^{+} \mathrm{BF}_{4}{ }^{-}$in ethylene carbonate, propylene carbonate, and dimethyl carbonate solvents, J. Phys. Chem. A 102 (1998) 1055-1061.

[25] J. Wang, R. M. Wolf, J. W. Caldwell, P. A. Kollman, D. A. Case, Development and testing of a general amber force field, J. Comput. Chem. 25 (9) (2004) 1157-1174.

[26] S. J. Keasler, S. M. Charan, C. D. Wick, I. G. Economou, J. I. Siepmann, Transferable potentials for phase equilibria-united atom description of five-and six-membered cyclic alkanes and ethers, J. Phys. Chem. B 116 (36) (2012) 11234-11246.

[27] W. L. Jorgensen, D. S. Maxwell, J. Tirado-Rives, Development and testing of the opls all-atom force field on conformational energetics and properties of organic liquids, J. Am. Chem. Soc 118 (45) (1996) 11225-11236.

[28] P. M. Anderson, M. R. Wilson, Developing a force field for simulation of poly (ethylene oxide) based upon $a b$ initio calculations of 1,2 dimethoxyethane, Mol. Phys 103 (1) (2005) 89-97.

[29] Z. Li, O. Borodin, G. D. Smith, D. Bedrov, Effect of organic solvents on $\mathrm{Li}^{+}$ion solvation and transport in ionic liquid electrolytes: A molecular dynamics simulation study, J. Phys. Chem. B 119 (7) (2015) 3085-3096.

[30] V. Lesch, Z. Li, D. Bedrov, O. Borodin, A. Heuer, The influence of cations on lithium ion coordination and transport in ionic liquid electrolytes: a md simulation study, Phys. Chem. Chem. Phys. 18 (1) (2016) 382-392.

[31] O. Borodin, G. D. Smith, LiTFSI structure and transport in ethylene carbonate from molecular dynamics simulations, J. Phys. Chem. B 110 (2006) 4971-4977.

[32] D. M. Seo, P. D. Boyle, R. D. Sommer, J. S. Daubert, O. Borodin, W. A. Henderson, Solvate structures and spectroscopic characterization of LiTFSI electrolytes, J. Phys. Chem. B 118 (47) (2014) 13601-13608.

[33] O. Borodin, G. D. Smith, Development of many- body polarizable force fields for Li-battery applications: 2. LiTFSI-doped oligoether, polyether, and carbonate-based electrolytes, J. Phys. Chem. B 110 (12) (2006) 62936299

[34] O. Borodin, G. Smith, $\mathrm{Li}^{+}$transport mechanism in oligo (ethylene oxide) s compared to carbonates, J. Solution Chem. 36 (6) (2007) 803-813.

[35] N. S. Barbosa, Y. Zhang, E. R. Lima, F. W. Tavares, E. J. Maginn, Development of an amber-compatible transferable force field for poly (ethylene glycol) ethers (glymes), J. Mol. Model 23 (6) (2017) 194.

[36] H. Farber, S. Petrucci, Kinetics of complexation of 18-crown-6 ether with lithium perchlorate in solvents of low permittivity. 1, 3-dioxolane and 1, 2-dimethoxyethane, J. Phys. Chem. 85 (10) (1981) 1396-1401.

[37] B. B. Gurung, M. N. Roy, Study of densities, viscosities and ultrasonic speeds of binary mixtures containing 1,2-dimethoxyethane and an alkan1-ol at 298.15 K, J. Solution Chem. 35 (12) (2006) 1587-1606.

[38] B. Giner, H. Artigas, M. Haro, C. Lafuente, M. López, Viscosities of binary mixtures of 1, 3-dioxolane or 1, 4-dioxane with isomeric chlorobutanes, J. Mol. Liq. 129 (3) (2006) 176-180.
[39] K. Hayamizu, Y. Aihara, S. Arai, C. G. Martinez, Pulse-gradient spinecho ${ }^{1} \mathrm{H},{ }^{7} \mathrm{Li}$, and ${ }^{19} \mathrm{~F}$ NMR diffusion and ionic conductivity measurements of 14 organic electrolytes containing $\mathrm{LiN}\left(\mathrm{SO}_{2} \mathrm{CF}_{3}\right)_{2}$, J. Phys. Chem. B 103 (3) (1999) 519-524.

[40] D. van der Spoel, E. Lindahl, B. Hess, G. Groenhof, A. E. Mark, H. J. Berendsen, GROMACS: fast, flexible, and free, J. Comput. Chem. 26 (16) (2005) 1701-1718.

[41] H. J. Berendsen, J. v. Postma, W. F. van Gunsteren, A. DiNola, J. Haak, Molecular dynamics with coupling to an external bath, J. Chem. Phys. 81 (8) (1984) 3684-3690.

[42] M. Parrinello, A. Rahman, Crystal structure and pair potentials: A molecular-dynamics study, Phys. Rev. Lett. 45 (14) (1980) 1196.

[43] M. Parrinello, A. Rahman, Polymorphic transitions in single crystals: A new molecular dynamics method, J. Appl. Phys. 52 (12) (1981) 7182 7190 .

[44] T. Darden, D. York, L. Pedersen, Particle mesh ewald: An n log (N) method for ewald sums in large systems, J. Chem. Phys. 98 (12) (1993) 10089-10092.

[45] U. Essmann, L. Perera, M. L. Berkowitz, T. Darden, H. Lee, L. G. Pedersen, A smooth particle mesh ewald method, J. Chem. Phys. 103 (19) (1995) 8577-8593.

[46] B. Hess, H. Bekker, H. J. Berendsen, J. G. Fraaije, et al., LINCS: a linear constraint solver for molecular simulations, J. Comput. Chem. 18 (12) (1997) 1463-1472.

[47] B. Hess, P-LINCS: A parallel linear constraint solver for molecular simulation, J. Chem. Theory Comput. 4 (1) (2008) 116-122.

[48] I. V. Leontyev, A. A. Stuchebrukhov, Polarizable mean-field model of water for biological simulations with amber and charmm force fields, J. Chem. Theory Comput. 8 (9) (2012) 3207.

[49] I. Leontyev, A. Stuchebrukhov, Electronic polarizability and the effective pair potentials of water, J. Chem. Theory Comput. 6 (10) (2010) 3153.

[50] Z. Kann, J. Skinner, A scaled-ionic-charge simulation model that reproduces enhanced and suppressed water diffusion in aqueous salt solutions, J. Chem. Phys. 141 (10) (2014) 104507.

[51] Y. Marcus, The properties of solvents, Vol. 16, Wiley Chichester, 1998

[52] J. N. Canongia Lopes, A. A. Pádua, Molecular force field for ionic liquids composed of triflate or bistriflylimide anions, J. Phys. Chem. B 108 (43) (2004) 16893-16898.

[53] T. Méndez-Morales, J. Carrete, O. Cabeza, O. Russina, A. Triolo, L. J. Gallego, L. M. Varela, Solvation of lithium salts in protic ionic liquids: a molecular dynamics study, J. Phys. Chem. B 118 (3) (2014) 761-770

[54] L. X. Dang, Development of nonadditive intermolecular potentials using molecular dynamics: solvation of $\mathrm{Li}^{+}$and $\mathrm{F}^{-}$ions in polarizable water, $\mathrm{J}$. Chem. Phys. 96 (9) (1992) 6970-6977.

[55] S. Risse, C. J. Jafta, Y. Yang, N. Kardjilov, A. Hilger, I. Manke, M. Ballauff, Multidimensional operando analysis of macroscopic structure evolution in lithium sulfur cells by X-ray radiography, Phys. Chem. Chem. Phys. 18 (15) (2016) 10630-10636.

[56] M. Safari, C. Y. Kwok, L. F. Nazar, Transport properties of polysulfide species in lithium-sulfur battery electrolytes: Coupling of experiment and theory, ACS Cent Sci 2 (2016) 560-568.

[57] S. Lee, S. S. Park, Dielectric constants of binary mixtures of propylene carbonate with dimethyl carbonate and ethylene carbonate from molecular dynamics simulation: comparison between non-polarizable and polarizable force fields, Mol. Phys 111 (2) (2013) 277-285.

[58] M. Neumann, O. Steinhauser, On the calculation of the frequencydependent dielectric constant in computer simulations, Chem. Phys. Lett. 102 (6) (1983) 508-513.

[59] M. Neumann, Dipole moment fluctuation formulas in computer simulations of polar systems, Mol. Phys 50 (4) (1983) 841-858

[60] M. Neumann, O. Steinhauser, G. S. Pawley, Consistent calculation of the static and frequency-dependent dielectric constant in computer simulations, Mol. Phys 52 (1) (1984) 97-113.

[61] I. Leontyev, A. Stuchebrukhov, Accounting for electronic polarization in non-polarizable force fields, Phys. Chem. Chem. Phys. 13 (7) (2011) 2613-2626.

[62] B. J. Palmer, Transverse-current autocorrelation-function calculations of the shear viscosity for molecular liquids, Phys. Rev. E 49 (1) (1994) 359.

[63] M. J. Abraham, D. van der Spoel, E. Lindahl, B. Hess, GROMACS User Manual version 5.1. 2 (2016)

[64] B. Hess, Determining the shear viscosity of model liquids from molecular 
dynamics simulations, J. Chem. Phys. 116 (1) (2002) 209-217.

[65] R. Fort, W. Moore, Viscosities of binary liquid mixtures, Trans. Faraday Soc. 62 (1966) 1112-1119.

[66] I.-C. Yeh, G. Hummer, System-size dependence of diffusion coefficients and viscosities from molecular dynamics simulations with periodic boundary conditions, J. Phys. Chem. B 108 (40) (2004) 15873-15879.

[67] I.-C. Yeh, G. Hummer, Diffusion and electrophoretic mobility of singlestranded RNA from molecular dynamics simulations, Biophys. J. 86 (2) (2004) 681-689.

[68] M. Chintapalli, K. Timachova, K. R. Olson, S. J. Mecha, D. Devaux, J. M. DeSimone, N. P. Balsara, Relationship between conductivity, ion diffusion, and transference number in perfluoropolyether electrolytes, Macromolecules 49 (2016) 3508-3515.

[69] J.-P. Hansen, I. R. McDonald, Theory of simple liquids, Elsevier, 1990.

[70] D. Brouillette, D. E. Irish, N. J. Taylor, G. Perron, M. Odziemkowski, J. E. Desnoyers, Stable solvates in solution of lithium bis (trifluoromethylsulfone) imide in glymes and other aprotic solvents: Phase diagrams, crystallography and raman spectroscopy, Phys. Chem. Chem. Phys. 4 (24) (2002) 6063-6071.

[71] W. A. Henderson, Glyme- lithium salt phase behavior, J. Phys. Chem. B 110 (26) (2006) 13177-13183.

[72] V. Chaban, Solvation of lithium ion in dimethoxyethane and propylene carbonate, Chem. Phys. Lett. 631 (2015) 1-5.

[73] F. Cataldo, A revision of the gutmann donor numbers of a series of phosphoramides including tepa, European Chemical Bulletin 4 (1-3) (2015) 92-97.

[74] J. Zheng, M. Gu, H. Chen, P. Meduri, M. H. Engelhard, J.-G. Zhang, J. Liu, J. Xiao, Ionic liquid-enhanced solid state electrolyte interface (SEI) for lithium-sulfur batteries, J. Mater. Chem. A 1 (29) (2013) 8464-8470.

[75] H.-S. Kim, C.-S. Jeong, Electrochemical properties of binary electrolytes for lithium-sulfur batteries, Bull. Korean Chem. Soc. 32 (10) (2011) 3682-3686.

[76] N.-N. Rajput, V. Murugesan, Y. Shin, K.-S. Han, K.-C. Lau, J. Chen, J. Liu, L.-A. Curtiss, K.-T. Mueller, K.-A. Persson, Elucidating the Solvation Structure and Dynamics of Lithium Polysulfides Resulting from Competitive Salt and Solvent Interactions, Chem. Mater. 29 (8) (2017) 3375-3379.

[77] V. Gutmann, Empirical parameters for donor and acceptor properties of solvents, Electrochim. Acta 21 (9) (1976) 661-670.

[78] C. Schröder, Comparing reduced partial charge models with polarizable simulations of ionic liquids, Phys. Chem. Chem. Phys. 14 (9) (2012) 3089-3102.

[79] L. Saiz, and E. Guardia, J. Padró, Dielectric properties of liquid ethanol. A computer simulation study, J. Chem. Phys. 113 (7) (2000) 2814-2822.

[80] W. D. Cornell, P. Cieplak, C. .I. Bayly, I. R. Gould, K. M. Merz, D. M. Ferguson, D. C. Spellmeyer, T. Fox, J. W. Caldwell, P. A. Kollman, A second generation force field for the simulation of proteins, nucleic acids, and organic molecules, J. Am. Chem. Soc. 117 (19) (1995) 5179-5197

[81] K. Ueno, K. Yoshida, M. Tsuchiya, T. Mizuho, N. Tachikawa, K. Dokko, M. Watanabe, Glyme-lithium salt equimolar molten mixtures: concentrated solutions or solvate ionic liquids?, J. Phys. Chem. B 116 (36) (2012) $11323-11331$ 